\title{
A parallel algorithm for discrete least squares rational approximation
}

\author{
Marc Van Barel and Adhemar Bultheel \\ Department of Computer Science \\ K.U. Leuven
}

\begin{abstract}
A new method for discrete least squares linearized rational approximation is presented. It generalizes the algorithm of Rutishauser-Gragg-Harrod-Reichel for discrete least squares polynomial approximation to the rational case. The algorithm is fast in the sense that it requires order $m \alpha$ computation time where $m$ is the number of data points and $\alpha$ is the degree of the approximant. We describe how this algorithm can be implemented in parallel.
\end{abstract}

\section{Introduction}

In our previous publications $[10,11]$, we investigated the rational interpolation problem not only for scalar function values but also for the vector case. The standpoint we took was a theoretical one. We found a parametrization of all solutions of the rational interpolation problem and fast algorithms to compute this parametrization. Fast means of order $m^{2}$ floating point operations (flops) if there are $m$ interpolation data. However, the algorithms which we designed are not numerically stable. This limits their applicability to theoretical investigation of the rational interpolation problem or to those cases where there are no round-off errors, e.g. when the computations are done within a finite field.

In this paper we replace the interpolation conditions by a discrete least squares criterion and describe an algorithm to solve this problem. It generalizes the algorithm of Reichel [6] for the polynomial case. The latter is inspired by the Rutishauser-Gragg-Harrod algorithm [8, 5, 1] for the computation of Jacobi matrices. A similar way of reasoning was followed by Golub and others [3,4]. It will turn out that our algorithm is fast, more specifically it is of order $m \alpha$ flops where $\alpha$ indicates the degree of the rational approximant. Implementing this algorithm in a pipelined fashion on $\alpha$ processors working in parallel reduces the computational time to order $m$.

\section{The theory behind the algorithm}

Let us assume that the function values are given in the form $f_{i} / e_{i}$ for the abscissae $z_{i}, i=$ $1,2, \ldots, m$, where $f_{i}, e_{i}$ and $z_{i}$ are all real numbers. If we define the degree of a rational form $n(z) / d(z)$ with $(n, d) \neq(0,0)$ as $\operatorname{deg}(n(z), d(z))=\max \{\operatorname{deg} n(z), \operatorname{deg} d(z)\}$, then in the rational interpolation problem, one wants to describe all polynomial couples $(n(z), d(z))$ 
of minimal degree which satisfy the interpolation conditions

$$
\frac{n\left(z_{i}\right)}{d\left(z_{i}\right)}=\frac{f_{i}}{e_{i}}, i=1,2, \ldots, m
$$

In [10], a parametrization was given and an efficient algorithm to solve this problem. When the data are corrupted with noise, one does not want the interpolation conditions to be satisfied exactly. We rather minimize the norm of the linearized residual vector with components

$$
r_{i}=f_{i} d\left(z_{i}\right)-e_{i} n\left(z_{i}\right), i=1,2, \ldots, m .
$$

We shall fix the degree of the approximant $n(z) / d(z)$ to be $\alpha=\max \{\operatorname{deg} n(z), \operatorname{deg} d(z)\}$ where usually $\alpha \ll m$. We also normalize the approximant in the following sense. Suppose $n(z)=n_{0}+n_{1} z+\cdots+n_{\alpha} z^{\alpha}$ and $d(z)=d_{0}+d_{1} z+\cdots+d_{\alpha} z^{\alpha}$, then we require $n_{\alpha}=1$ if $\operatorname{deg} n(z)=\alpha$ and $d_{\alpha}=1$ if $\operatorname{deg} n(z)<\alpha$ (and $\operatorname{deg} d(z)=\alpha$ ). Note that we have given preference to the numerator polynomial in the normalization procedure. However, by switching the role of $f_{i}$ and $e_{i}$ and of $n(z)$ and $d(z)$, we get the symmetric problem setting giving preference to $d(z)$ in the normalization procedure. Thus in this paper, we shall solve the following rational approximation problem.

Definition 2.1 (rational approximation problem) Given the data points $z_{i}$ with corresponding estimated function values $f_{i} / e_{i}, i=1,2, \ldots, m$, given the degree $\alpha$ and the weights $w_{i}>0, i=1,2, \ldots, m$, we look for the normalized rational form $n(z) / d(z)$ of degree $\alpha$ satisfying the following least squares approximation criterion:

$$
\text { minimize } \operatorname{dist}^{2}(n, d)=\sum_{i=1}^{m} w_{i}\left(e_{i} n\left(z_{i}\right)-f_{i} d\left(z_{i}\right)\right)^{2}=\sum_{i=1}^{m} w_{i} r_{i}^{2},
$$

where $\operatorname{dist}(n, d)$ denotes the distance between the rational form $(n(z), d(z))$ and the data.

The least squares problem can be reformulated as a block problem, i.e. we should minimize

$$
\sum_{i=1}^{m}\left[d\left(z_{i}\right) n\left(z_{i}\right)\right] v_{i}\left[\begin{array}{l}
d\left(z_{i}\right) \\
n\left(z_{i}\right)
\end{array}\right]
$$

with

$$
v_{i}=\left[\begin{array}{c}
f_{i}^{\prime} \\
-e_{i}^{\prime}
\end{array}\right]\left[f_{i}^{\prime}-e_{i}^{\prime}\right] \text { and } e_{i}^{\prime}=\sqrt{w_{i}} e_{i} \text { and } f_{i}^{\prime}=\sqrt{w_{i}} f_{i} .
$$

Denoting the approximant as $a(z)=[d(z) n(z)]^{T}$, this can be formulated as

$$
\sum_{i=1}^{m} w_{i} r_{i}^{2}=\|a(z)\|_{v}^{2}
$$

with

$$
\|a(z)\|_{v}^{2}=\langle a(z), a(z)\rangle_{v}
$$

and

$$
\langle a(z), b(z)\rangle_{v}=\sum_{i=1}^{m} a\left(z_{i}\right)^{T} v_{i} b\left(z_{i}\right)
$$

for any $a(z)$, and $b(z)$ polynomial vectors in $\mathbb{R}[z]^{2}$. We also need a matrix version. So, if $A(z), B(z) \in \mathbb{R}[z]^{2 \times 2}$, we define

$$
\langle A(z), B(z)\rangle_{v}=\sum_{i=1}^{m} A\left(z_{i}\right)^{T} v_{i} B\left(z_{i}\right)
$$


Now suppose that we can construct some orthonormal polynomials $\left\{\phi_{0}, \phi_{1}, \ldots, \phi_{m}\right\}$ in $\mathbb{R}[z]^{2 \times 2}$, such that $\phi_{k}(z)=\phi_{k k} z^{k}+\cdots+\phi_{k 0}$ with $\phi_{k k}$ regular and such that

$$
\left\langle\phi_{i}(z), \phi_{j}(z)\right\rangle_{v}=\delta_{i j} I_{2}
$$

with $I_{2}$ the $2 \times 2$ unit matrix. Then we can express $a(z)$ as a linear combination

$$
a(z)=\sum_{i=0}^{\alpha} \phi_{i}(z) a_{i}, \quad a_{i} \in \mathbb{R}^{2}
$$

Thus,

$$
\begin{aligned}
\|a(z)\|_{v}^{2} & =\sum_{i=1}^{m} a\left(z_{i}\right)^{T} v_{i} a\left(z_{i}\right) \\
& =\sum_{i=1}^{m}\left(\sum_{k=0}^{\alpha} a_{k}^{T} \phi_{k}\left(z_{i}\right)^{T}\right) v_{i}\left(\sum_{l=0}^{\alpha} \phi_{l}\left(z_{i}\right) a_{l}\right) \\
& =\sum_{k, l=0}^{\alpha} a_{k}^{T}\left(\sum_{i=1}^{m} \phi_{k}\left(z_{i}\right)^{T} v_{i} \phi_{l}\left(z_{i}\right)\right) a_{l} \\
& =\sum_{k, l=0}^{\alpha} a_{k}^{T}\left\langle\phi_{k}, \phi_{l}\right\rangle_{v} a_{l} \\
& =\sum_{k=0}^{\alpha} a_{k}^{T} a_{k} .
\end{aligned}
$$

Because $a_{\alpha}$ can not be zero, but all $a_{k}, k=0,1, \ldots, \alpha-1$ can be chosen as zero, we find that for the minimizing $a(z)$

$$
a(z)=\sum_{k=0}^{\alpha} \phi_{k}(z) a_{k}=\phi_{\alpha}(z) a_{\alpha}
$$

where $a_{\alpha}$ is chosen to minimize

$$
\|a\|_{v}^{2}=a_{\alpha}^{T} a_{\alpha}=a_{1 \alpha}^{2}+a_{2 \alpha}^{2} .
$$

Given the normalizing conditions for the approximant and the leading coefficient of $\phi_{\alpha}(z)$, this is an easy problem to solve.

Our first task will be to construct the orthogonal block polynomials $\phi_{j}$. This could be done using a block version of the Lanczos algorithm, but since Lanczos is numerically unstable, we use a generalization of the numerically better method introduced by Gragg et al. $[7,6]$ to compute a polynomial approximation in the least squares sense.

\section{The algorithm}

The algorithm starts by applying some orthogonal similarity transformations on the data which are first arranged in an array as follows

$$
M=\left[\begin{array}{c|c}
0 & {\left[f^{\prime}-e^{\prime}\right]^{T}} \\
\left.\hline f^{\prime}-e^{\prime}\right] & Z
\end{array}\right]
$$


where we used the notation

$$
\begin{aligned}
{\left[\begin{array}{ll}
f^{\prime} & -e^{\prime}
\end{array}\right] } & =\left[\begin{array}{cccc}
f_{1}^{\prime} & f_{2}^{\prime} & \cdots & f_{m}^{\prime} \\
-e_{1}^{\prime} & -e_{2}^{\prime} & \cdots & -e_{m}^{\prime}
\end{array}\right]^{T}, \\
Z & =\operatorname{diag}\left(z_{1}, z_{2}, \ldots, z_{m}\right) .
\end{aligned}
$$

We have a double column in the SW quadrant, which is reflected as transposed rows in the NE quadrant, and in the SE quadrant we originally find the diagonal matrix of abscissae. We now apply Givens reflections (we could also use Givens rotations) to the left and right of this initial matrix $M$ to transform it into $Q^{\prime T} M Q^{\prime}$, a block upper Hessenberg matrix (the blocks are two by two), where $Q^{\prime}=I_{2} \oplus Q$. Because $Z$ is diagonal, we get

$$
\left(Q^{T} Z Q\right)^{T}=Q^{T} Z Q
$$

Therefore, $Q^{T} Z Q$ will be symmetric block tridiagonal. More precisely, $Q^{T} Z Q$ will be symmetric pentadiagonal which is also two by two block tridiagonal. It is easy to get similar results when all points $z_{i}, i=1,2, \ldots, m$ are purely imaginary. When the data points lie on the unit circle, Gragg et al. [7] solve the least squares polynomial approximation problem using a Schur parametrization of the unitary Hessenberg matrix that plays the central role in the theory. We shall treat the rational unit circle problem in another publication.

Let us return to the computational scheme and explain with an example how the transformation $Q^{\prime T} M Q^{\prime}$ is actually performed. The technical details are descibed in section 3 . Entries which are not necessarily zero, are denoted by $\times$. By $\odot$, we denote the two entries determining the Givens reflection making one of the two elements equal to zero. The entries which will change when applying this Givens reflection are denoted by $\otimes$. These include the zero entries which could get a nonzero value after the Givens reflection is applied (fill-in). Let us rewrite the initial setup using this new notation where we have already indicated which two elements determine the first Givens reflection that will be applied to the left. In our example, we have chosen $m=8$.

\begin{tabular}{cc|cccccccc} 
& & $\times$ & $\times$ & $\times$ & $\times$ & $\times$ & $\times$ & $\times$ & $\times$ \\
& & $\times$ & $\times$ & $\times$ & $\times$ & $\times$ & $\times$ & $\times$ & $\times$ \\
\hline$\odot$ & $\times$ & $\times$ & & & & & & & \\
$\odot$ & $\times$ & & $\times$ & & & & & & \\
$\times$ & $\times$ & & & $\times$ & & & & & \\
$\times$ & $\times$ & & & & $\times$ & & & & \\
$\times$ & $\times$ & & & & & $\times$ & & & \\
$\times$ & $\times$ & & & & & & $\times$ & & \\
$\times$ & $\times$ & & & & & & & $\times$ & \\
$\times$ & $\times$ & & & & & & & & $\times$
\end{tabular}

Applying the Givens reflection to the left of this scheme, generates the following fill-in.

\begin{tabular}{cc|cccccccc} 
& & $\times$ & $\times$ & $\times$ & $\times$ & $\times$ & $\times$ & $\times$ & $\times$ \\
& & $\times$ & $\times$ & $\times$ & $\times$ & $\times$ & $\times$ & $\times$ & $\times$ \\
\hline$\otimes$ & $\otimes$ & $\otimes$ & $\otimes$ & & & & & & \\
0 & $\otimes$ & $\otimes$ & $\otimes$ & & & & & & \\
$\times$ & $\times$ & & & $\times$ & & & & & \\
$\times$ & $\times$ & & & & $\times$ & & & & \\
$\times$ & $\times$ & & & & & $\times$ & & & \\
$\times$ & $\times$ & & & & & & $\times$ & & \\
$\times$ & $\times$ & & & & & & & $\times$ & \\
$\times$ & $\times$ & & & & & & & & $\times$
\end{tabular}


Applying this Givens reflection to the right generates a zero in the top row for reasons of symmetry. In the next scheme, we introduce the fill-in introduced by this operation and we indicate the two elements which will determine the next Givens reflection.

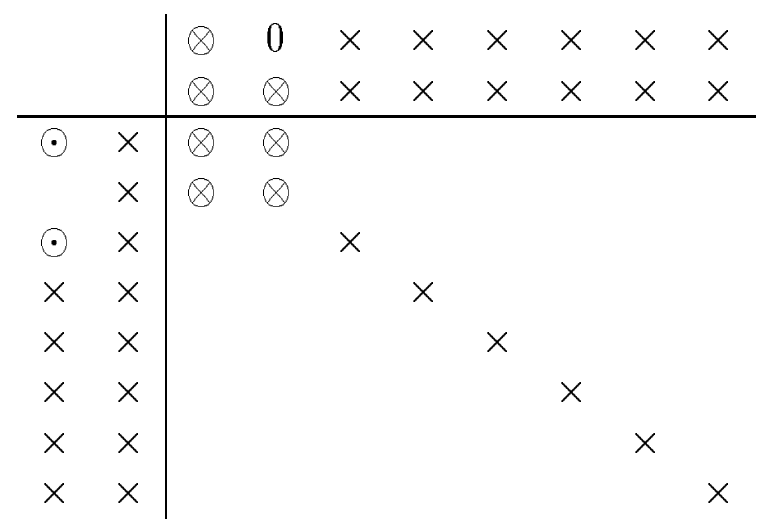

Transforming the third row of the lower block of the computational scheme leads to the following table.

\begin{tabular}{cc|cccccccc} 
& & $\times$ & & $\times$ & $\times$ & $\times$ & $\times$ & $\times$ & $\times$ \\
& & $\times$ & $\times$ & $\times$ & $\times$ & $\times$ & $\times$ & $\times$ & $\times$ \\
\hline$\otimes$ & $\otimes$ & $\otimes$ & $\otimes$ & $\otimes$ & & & & & \\
& $\times$ & $\times$ & $\times$ & & & & & & \\
0 & $\otimes$ & $\otimes$ & $\otimes$ & $\otimes$ & & & & & \\
$\times$ & $\times$ & & & & $\times$ & & & & \\
$\times$ & $\times$ & & & & & $\times$ & & & \\
$\times$ & $\times$ & & & & & & $\times$ & & \\
$\times$ & $\times$ & & & & & & & $\times$ & \\
$\times$ & $\times$ & & & & & & & & $\times$
\end{tabular}

Apply the Givens reflection to the right and indicate the two elements determining the next reflection, and you get the following table.

\begin{tabular}{cc|cccccccc} 
& & $\otimes$ & & 0 & $\times$ & $\times$ & $\times$ & $\times$ & $\times$ \\
& & $\otimes$ & $\times$ & $\otimes$ & $\times$ & $\times$ & $\times$ & $\times$ & $\times$ \\
\hline$\times$ & $\times$ & $\otimes$ & $\times$ & $\otimes$ & & & & & \\
& $\odot$ & $\otimes$ & $\times$ & $\otimes$ & & & & & \\
& $\odot$ & $\otimes$ & $\times$ & $\otimes$ & & & & & \\
$\times$ & $\times$ & & & & $\times$ & & & & \\
$\times$ & $\times$ & & & & & $\times$ & & & \\
$\times$ & $\times$ & & & & & & $\times$ & & \\
$\times$ & $\times$ & & & & & & & $\times$ & \\
$\times$ & $\times$ & & & & & & & & $\times$
\end{tabular}

Application of the next Givens reflection to the left and right, leads to the following scheme. 


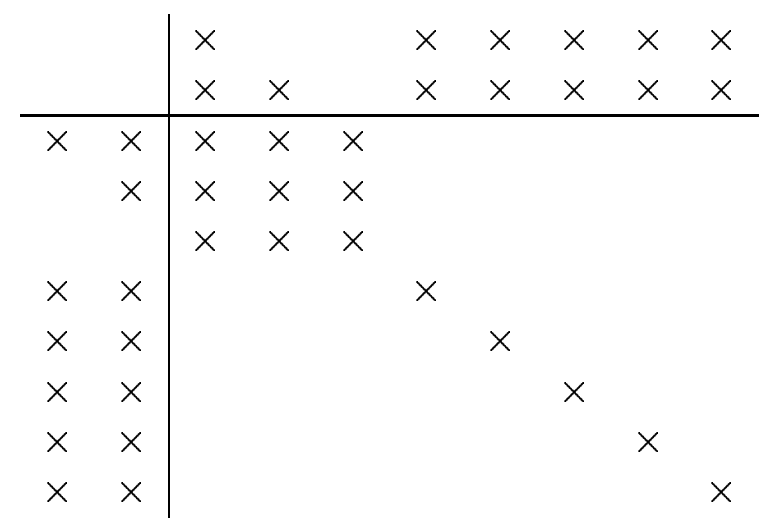

It is clear that the upper left part has become a pentadiagonal matrix. Following the same path of computation for the following rows (and symmetrically for the columns) except for the last one, the scheme eventually becomes as follows.

\begin{tabular}{cc|cccccccc} 
& & $\times$ & & & & & & & $\times$ \\
& $\times$ & $\times$ & & & & & & $\times$ \\
\hline & $\times$ & $\times$ & $\times$ & $\times$ & & & & & \\
& $\times$ & $\times$ & $\times$ & $\times$ & & & & \\
& & $\times$ & $\times$ & $\times$ & $\times$ & $\times$ & & & \\
& & $\times$ & $\times$ & $\times$ & $\times$ & $\times$ & & \\
& & & $\times$ & $\times$ & $\times$ & $\times$ & $\times$ & \\
& & & & $\times$ & $\times$ & $\times$ & $\times$ & \\
& & & & & $\times$ & $\times$ & $\times$ & \\
& & & & & & & & $\times$
\end{tabular}

We have already indicated the two elements determining the first Givens reflection to handle the last row (and column) of the table. Applying this Givens reflection to the left, leads us to the following result.

\begin{tabular}{cc|ccccccccc} 
& & $\times$ & & & & & & & $\times$ \\
& $\times$ & $\times$ & & & & & & $\times$ \\
\hline & $\otimes$ & $\otimes$ & $\otimes$ & $\otimes$ & & & & & $\otimes$ \\
& $\times$ & $\times$ & $\times$ & $\times$ & & & & \\
& & $\times$ & $\times$ & $\times$ & $\times$ & $\times$ & & & \\
& & $\times$ & $\times$ & $\times$ & $\times$ & $\times$ & & \\
& & & $\times$ & $\times$ & $\times$ & $\times$ & $\times$ & \\
& & & & $\times$ & $\times$ & $\times$ & $\times$ & \\
& & & & & & $\times$ & $\times$ & $\times$ & \\
0 & $\otimes$ & $\otimes$ & $\otimes$ & $\otimes$ & & & & & $\otimes$
\end{tabular}

Note that we have shifted the position of the first nonzero element of the last row one place to the right by applying the Givens reflection on vectors of length 6 . Similarly, by applying the Givens reflection to the right, the position of the first nonzero element of the last column will be shifted one place down again by applying the Givens reflection on vectors of length 6 . The resulting scheme looks as follows. 


\begin{tabular}{cc|cccccccc} 
& & $\otimes$ & & & & & & & 0 \\
\hline & $\otimes$ & $\times$ & & & & & & $\theta$ \\
\hline & $\odot$ & $\otimes$ & $\times$ & $\times$ & & & & & $\otimes$ \\
& & $\otimes$ & $\times$ & $\times$ & $\times$ & & & & $\otimes$ \\
& & & $\times$ & $\times$ & $\times$ & $\times$ & & & $\otimes$ \\
& & $\times$ & $\times$ & $\times$ & $\times$ & $\times$ & & \\
& & & $\times$ & $\times$ & $\times$ & $\times$ & $\times$ & \\
& & & & $\times$ & $\times$ & $\times$ & $\times$ & \\
& & & & & $\times$ & $\times$ & $\times$ & \\
& $\odot$ & $\times$ & $\times$ & & & & & $\otimes$
\end{tabular}

In this way we can shift the place of the four nonzero elements of the last row and of the last column in the direction of the diagonal by applying Givens reflections to vectors of length 6 . Note that the length of the vectors involved decreases when the diagonal is reached. Let us show the application of the last Givens reflection to the left and right of the computational scheme. Before we apply this reflection, the situation is as follows.

\begin{tabular}{cc|ccccccccc} 
& & $\times$ & & & & & & & \\
& & $\times$ & $\times$ & & & & & & \\
\hline & $\times$ & $\times$ & $\times$ & $\times$ & & & & & \\
& $\times$ & $\times$ & $\times$ & $\times$ & $\times$ & & & & \\
& & $\times$ & $\times$ & $\times$ & $\times$ & $\times$ & & & \\
& & $\times$ & $\times$ & $\times$ & $\times$ & $\times$ & & \\
& & $\times$ & $\times$ & $\times$ & $\times$ & $\times$ & $\times$ \\
& & & & $\times$ & $\times$ & $\times$ & $\times$ & $\times$ \\
& & & & & $\odot$ & $\times$ & $\times$ & $\times$ \\
& & & & & $\odot$ & $\times$ & $\times$ & $\times$
\end{tabular}

Applying the Givens reflection to the left and right involves vectors of length 4 and results in the following final pentadiagonal scheme.

\begin{tabular}{cc|ccccccccc} 
& & $\times$ & & & & & & & \\
& $\times$ & $\times$ & & & & & & \\
\hline$\times$ & $\times$ & $\times$ & $\times$ & $\times$ & & & & & \\
& $\times$ & $\times$ & $\times$ & $\times$ & $\times$ & & & & \\
& & $\times$ & $\times$ & $\times$ & $\times$ & $\times$ & & & \\
& $\times$ & $\times$ & $\times$ & $\times$ & $\times$ & & \\
& & $\times$ & $\times$ & $\times$ & $\times$ & $\times$ & \\
& & & $\times$ & $\times$ & $\times$ & $\times$ & $\times$ \\
& & & & & $\times$ & $\times$ & $\times$ & $\times$ \\
& & & & & & $\times$ & $\times$ & $\times$
\end{tabular}

It is clear that by keeping the sparse structure, the Givens reflections are applied to vectors of maximum length 6 . Therefore, the computational work for this part of the algorithm is of the order $m^{2}$. Note that the sequence in which these Givens reflections are applied is very important to keep the sparse structure. Hence, we can not use Householder transformations in this case. Note also that this transformation to a pentadiagonal form can always be carried out. One of the entries determining a Givens reflection could be zero, reducing the Givens reflection to a permutation. Even both entries could be zero. In this case, we could leave the situation unchanged by using the trivial Givens rotation

$$
\left[\begin{array}{ll}
1 & 0 \\
0 & 1
\end{array}\right] \text {. }
$$


or, when we choose only to use reflections, we could apply the trivial Givens reflection

$$
\left[\begin{array}{cc}
1 & 0 \\
0 & -1
\end{array}\right]
$$

A more detailed description of one step of the algorithm will be given in section 5 . We show first how our approximation problem can be solved.

\section{The solution of the rational approximation problem}

It turns out that the resulting pentadiagonal matrix, when considered as a $2 \times 2$ block tridiagonal actually gives the recurrence coefficients for the block orthogonal polynomials $\phi_{j}$ which we needed. So we divide this scheme in two by two blocks and denote these blocks as follows.

\begin{tabular}{c|ccccc} 
& $B_{0}^{T}$ & & & & \\
\hline \multirow{3}{*}{$B_{0}$} & $A_{1}$ & $B_{1}^{T}$ & & & \\
& $B_{1}$ & $A_{2}$ & $\ddots$ & & \\
& & $B_{2}$ & $\ddots$ & $\ddots$ & \\
& & & $\ddots$ & $A_{m^{\prime}-1}$ & $B_{m^{\prime}-1}^{T}$ \\
& & & & $B_{m^{\prime}-1}$ & $A_{m^{\prime}}$
\end{tabular}

The value of $m^{\prime}$ is equal to the integer part of $(m+1) / 2$. If $m$ is even, all blocks are two by two. However, if $m$ is odd, $A_{m^{\prime}}$ is a scalar, while $B_{m^{\prime}-1}$ is a row vector with two components. Note that all blocks $A_{j}, j=1,2, \ldots, m^{\prime}$ are symmetric. Without loss of generality, we shall assume in the sequel that $m$ is even. In this case, all $B_{j}, j=0,1, \ldots, m^{\prime}-1$ are right triangular, also the last one. For the moment we also assume that all $B_{j}$ are nonsingular, which we shall call the regular case. If some $B_{j}$ is singular, we call it the singular case.

\subsection{The regular case}

In this case we can define the following block polynomials, which play a crucial role in the solution of the rational approximation problem.

Definition 4.1 (polynomials $\phi_{j}$ ) The first two block polynomials $\phi_{-1}$ and $\phi_{0}$ of the sequence are defined as follows:

$$
\begin{aligned}
\phi_{-1} & =\left[\begin{array}{ll}
0 & 0 \\
0 & 0
\end{array}\right], \\
\phi_{0} & =B_{0}^{-1} .
\end{aligned}
$$

The next block polynomials $\phi_{j}(z), j=1,2,3, \ldots, m^{\prime}-1$ are defined by the following recurrence relation

$$
\phi_{j}(z)=\phi_{j-1}(z)\left[z I_{2}-A_{j}\right] B_{j}^{-1}-\phi_{j-2}(z) B_{j-1}^{T} B_{j}^{-1}, \quad j=1,2, \ldots, m^{\prime}-1,
$$

based on the previous two block polynomials $\phi_{j-1}(z)$ and $\phi_{j-2}(z)$ and the two by two blocks of the block tridiagonal matrix $P$

$$
P=\operatorname{tridiag}\left[\begin{array}{cccc}
B_{1}^{T} & B_{2}^{T} & \cdots & B_{m^{\prime}-1}^{T} \\
A_{1} & A_{2} & \cdots & A_{m^{\prime}} \\
B_{1} & B_{2} & \cdots & B_{m^{\prime}-1}
\end{array}\right],
$$

which has the properties described above. 
We shall derive some important properties for these block polynomials $\phi_{j}(z)$.

Property 4.1 (degree, leading coefficient) The block polynomial $\phi_{j}(z), j=0,1, \ldots, m^{\prime}-$ 1 has degree $j$ and the highest degree coefficient is equal to the right triangular matrix $B_{0}^{-1} B_{1}^{-1} \cdots B_{j}^{-1}$ if all $B_{j}$ are regular.

Proof. The property is certainly true for $\phi_{0}$. From the recurrence relation, an induction proof follows readily.

The next property will indicate the relationship between the block polynomials $\phi_{j}$ and the unitary (orthogonal) matrix $Q$ representing the Givens reflections (rotations) applied to the right of our computational scheme, i.e. $Q^{T} Z Q=P$ and $Q^{T}\left[f^{\prime},-e^{\prime}\right]=\left[B_{0}^{T}, 0, \ldots, 0\right]^{T}$.

Property 4.2 (the values $\phi_{j}\left(z_{i}\right)$ ) Assume all the $B_{j}$ are regular, then the orthogonal matrix $Q$ is directly connected to the function values of the block polynomials $\phi_{j}$ in the data points,

$$
Q=D\left[\begin{array}{cccc}
\phi_{0}\left(z_{1}\right) & \phi_{1}\left(z_{1}\right) & \cdots & \phi_{m^{\prime}-1}\left(z_{1}\right) \\
\phi_{0}\left(z_{2}\right) & \phi_{1}\left(z_{2}\right) & \cdots & \phi_{m^{\prime}-1}\left(z_{2}\right) \\
\vdots & \vdots & & \vdots \\
\phi_{0}\left(z_{m}\right) & \phi_{1}\left(z_{m}\right) & \cdots & \phi_{m^{\prime}-1}\left(z_{m}\right)
\end{array}\right]
$$

with $D$ the block diagonal matrix

$$
D=\operatorname{diag}\left\{\left[f_{1}^{\prime},-e_{1}^{\prime}\right],\left[f_{2}^{\prime},-e_{2}^{\prime}\right], \ldots,\left[f_{m}^{\prime},-e_{m}^{\prime}\right]\right\} .
$$

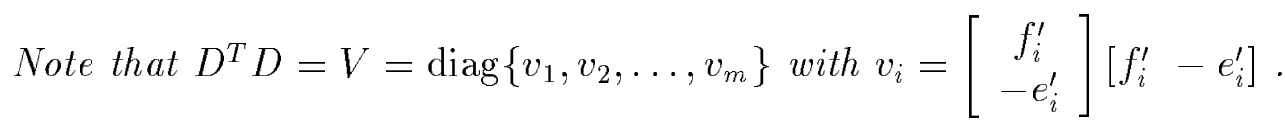

Proof. We partition the orthogonal matrix $Q$ in block columns where each block column consists of two simple columns, i.e. $Q=\left[Q_{1}, Q_{2}, \ldots, Q_{m^{\prime}}\right]$. From the relation

$$
Q^{T}\left[\begin{array}{ll}
f^{\prime} & -e^{\prime}
\end{array}\right]=\left[\begin{array}{c}
B_{0} \\
0 \\
\vdots \\
0
\end{array}\right]=\left[\begin{array}{c}
\phi_{0}^{-1} \\
0 \\
\vdots \\
0
\end{array}\right]
$$

it follows that the property is true for the first block column of $Q: Q_{1}=\left[f^{\prime},-e^{\prime}\right] \phi_{0}$. To prove the validity of the property for the remaining block columns $Q_{j}, j=2,3, \ldots, m^{\prime}$, we use the relationship $Z Q=Q P$ or

$$
z_{i} Q_{i, j-1}=Q_{i, j-2} B_{j-2}^{T}+Q_{i, j-1} A_{j-1}+Q_{i, j} B_{j-1}, \quad i=1,2, \ldots, m,
$$

where $Q_{i, j}$ denotes the $i$ th row of the block column $Q_{j}$ and $Q_{0}=0$. Hence, the $j$ th block column $Q_{j}$ can be written in terms of the two previous block columns as follows

$$
Q_{i, j}=Q_{i, j-1}\left(z_{i} I_{2}-A_{j-1}\right) B_{j-1}^{-1}-Q_{i, j-2} B_{j-2}^{T} B_{j-1}^{-1} .
$$

If we assume that the property is true for the previous block columns, we can prove that the property is also valid for the $j$ th block column by applying the property for the block columns $Q_{j-1}$ and $Q_{j-2}$ resulting in

$$
Q_{i, j}=\left[f_{i}^{\prime},-e_{i}^{\prime}\right]\left(\phi_{j-2}\left(z_{i}\right)\left(z_{i} I_{2}-A_{j-1}\right) B_{j-1}^{-1}-\phi_{j-3}\left(z_{i}\right) B_{j-2}^{T} B_{j-1}^{-1}\right) .
$$


Using the recurrence relation for the block polynomials $\phi_{j}$, we obtain that

$$
Q_{i, j}=\left[f_{i}^{\prime},-e_{i}^{\prime}\right] \phi_{j-1}\left(z_{i}\right),
$$

and this proves the property by induction on $j$.

The key to the solution of the rational approximation problem is the following orthogonality property of the block polynomials $\phi_{j}$.

Property 4.3 (orthonormality) The block polynomials $\phi_{j}$ are orthonormal in the following sense

$$
\begin{aligned}
& \sum_{i=1}^{m} \phi_{k}^{T}\left(z_{i}\right)\left[\begin{array}{c}
f_{i}^{\prime} \\
-e_{i}^{\prime}
\end{array}\right]\left[\begin{array}{ll}
f_{i}^{\prime} & -e_{i}^{\prime}
\end{array}\right] \phi_{l}\left(z_{i}\right)=\left[\begin{array}{ll}
0 & 0 \\
0 & 0
\end{array}\right], \quad \text { if } k \neq l \\
& =\left[\begin{array}{ll}
1 & 0 \\
0 & 1
\end{array}\right], \quad \text { if } k=l .
\end{aligned}
$$

Proof. From the previous property, we have the following relationship between the function value of the block polynomials $\phi_{j}$ in the points $z_{i}$ :

$$
Q_{i, j}=\left[f_{i}^{\prime},-e_{i}^{\prime}\right] \phi_{j-1}\left(z_{i}\right) .
$$

From the orthogonality of the block columns of $Q$, the property follows immediately.

Let us now return to the rational approximation problem. To solve this problem, we shall write the solution in terms of the orthonormal block polynomials $\phi_{j}$. Because the highest degree coefficients of these block polynomials are right triangular, we can write our solution $n(z) / d(z)$ of degree $\alpha$ always as follows

$$
\left[\begin{array}{l}
d(z) \\
n(z)
\end{array}\right]=\sum_{j=0}^{\alpha} \phi_{j}(z)\left[\begin{array}{l}
\mu_{j} \\
\nu_{j}
\end{array}\right]
$$

with $\left(\mu_{\alpha}, \nu_{\alpha}\right) \neq(0,0)$. Remember that the solution we are looking for, has to be normalized. From property 4.1, we know that the highest degree coefficient of $\phi_{\alpha}$ is equal to

$$
B_{0}^{-1} B_{1}^{-1} \cdots B_{\alpha}^{-1}=\left[\begin{array}{cc}
1 / \prod_{j=0}^{\alpha} b_{1, j} & \times \\
0 & 1 / \prod_{j=1}^{\alpha} b_{2, j}
\end{array}\right], \quad \text { if } B_{j}=\left[\begin{array}{cc}
b_{1, j} & b_{3, j} \\
0 & b_{2, j}
\end{array}\right] .
$$

The specific value of $\times$ is not important for the moment. To normalize $n(z) / d(z)$, we have to choose $\nu_{\alpha}=\prod_{j=0}^{\alpha} b_{2, j}$ if $n_{\alpha} \neq 0$ or $\mu_{\alpha}=\prod_{j=0}^{\alpha} b_{1, j}$ if $n_{\alpha}=0$. To solve the rational approximation problem, we have to minimize

$$
\sum_{i=1}^{m}\left(f_{i}^{\prime} d\left(z_{i}\right)-e_{i}^{\prime} n\left(z_{i}\right)\right)^{2} .
$$

Writing $n(z)$ and $d(z)$ in terms of the block polynomials $\phi_{j}$, this is equivalent to minimizing

$$
\sum_{i=1}^{m}\left(\sum_{k=0}^{\alpha}\left[\begin{array}{ll}
\mu_{k} & \nu_{k}
\end{array}\right] \phi_{k}^{T}\left(z_{i}\right)\right)\left[\begin{array}{c}
f_{i}^{\prime} \\
-e_{i}^{\prime}
\end{array}\right]\left[\begin{array}{ll}
f_{i}^{\prime} & -e_{i}^{\prime}
\end{array}\right]\left(\sum_{l=0}^{\alpha} \phi_{l}\left(z_{i}\right)\left[\begin{array}{l}
\mu_{l} \\
\nu_{l}
\end{array}\right]\right) .
$$

Using the orthogonality property 4.3 for the block polynomials $\phi_{j}(z)$, this simplifies to minimizing

$$
\sum_{k=0}^{\alpha}\left(\mu_{k}^{2}+\nu_{k}^{2}\right)
$$


First of all, we can make this entity smaller by putting $\mu_{k}$ and $\nu_{k}$ equal to zero for $k=$ $0,1, \ldots, \alpha-1$. This reduces the distance between $(n(z), d(z))$ and the data to $\sqrt{\mu_{\alpha}^{2}+\nu_{\alpha}^{2}}$. The way in which we have stated the rational approximation problem requires $n(z) / d(z)$ to have degree $\alpha$. From the normalization conditions, it is clear that we should therefore take the "right" structure, i.e.

$$
\nu_{\alpha}=\prod_{j=0}^{\alpha} b_{2, j} \text { and } \mu_{\alpha}=0, \quad \text { if } \prod_{j=0}^{\alpha} b_{2, j} \leq \prod_{j=0}^{\alpha} b_{1, j}
$$

and the "left" structure, i.e.

$$
\mu_{\alpha}=\prod_{j=0}^{\alpha} b_{1, j} \text { and } \nu_{\alpha}=0, \quad \text { if } \prod_{j=0}^{\alpha} b_{2, j} \geq \prod_{j=0}^{\alpha} b_{1, j}
$$

where, in case of equality of the two distances, we can choose between the two solutions. Note that this choice corresponds to our problem setting, but that we have the possibility to solve also slightly different problems in an equally simple way. E.g., we could have required $n(z) / d(z)$ to be strictly proper, i.e. with $n_{\alpha}=0$ and $d_{\alpha}=1$. Then we had to choose the second solution, i.e. the one with $\nu_{\alpha}=0$. It is easy to generalize this to more complicated structures than the strictly proper one. Suppose for example that we look for a solution with $n_{\alpha}=0, n_{\alpha-1}=0$ and $d_{\alpha}=1$. From $n_{\alpha}=0$, we easily derive that $\nu_{\alpha}=0$. From $d_{\alpha}=1$, it follows that $\mu_{\alpha}=\prod_{j=0}^{\alpha} b_{1, j}$. To make $n_{\alpha-1}$ equal to zero, we have to take $\nu_{\alpha-1}$ equal to a specific value, possibly different from zero. We shall not follow this path in the sequel but concentrate our attention on the original problem setting. The other cases are quite similar. Let us investigate how good the rational approximation is.

Theorem 4.4 If we write our rational approximant $n(z) / d(z)$ in terms of the parameters $\mu_{k}, \nu_{k}, k=0,1,2, \ldots, \alpha$, then the approximation error made in the $i$ th point $z_{i}, i=1,2, \ldots, m$ can be measured as follows

$$
f_{i}^{\prime} d\left(z_{i}\right)-e_{i}^{\prime} n\left(z_{i}\right)=\sum_{k=0}^{\alpha} Q_{i, k+1}\left[\begin{array}{l}
\mu_{k} \\
\nu_{k}
\end{array}\right] .
$$

Proof. This result follows immediately from the fact that

$$
\left[\begin{array}{ll}
f_{i}^{\prime} & -e_{i}^{\prime}
\end{array}\right] \phi_{k}\left(z_{i}\right)=Q_{i, k+1}
$$

For example, if we take the strictly proper solution, all $\mu_{k}$ and $\nu_{k}$ values are zero except one, $\mu_{\alpha}=\prod_{j=0}^{\alpha} b_{1, j}$. The approximation error in each point $z_{i}$ can thus be written as

$$
f_{i}^{\prime} d\left(z_{i}\right)-e_{i}^{\prime} n\left(z_{i}\right)=Q_{i, \alpha+1}\left[\begin{array}{c}
\mu_{\alpha} \\
0
\end{array}\right],
$$

i.e. the error is given by a scaled version of the first column of $Q_{\alpha+1}$.

\subsection{The singular case}

Until now, we have taken for granted that all the subdiagonal blocks $B_{j}$ were nonsingular ( $B_{m^{\prime}-1}$ has full rank). What will happen if one of the right triangular matrices $B_{j}$ is singular, 
i.e. if $b_{1, j}$ or $b_{2, j}$ is equal to zero? Without loss of generality, we shall assume that the first zero in the sequence $b_{1,0}, b_{2,0}, b_{1,1}, b_{2,1}, \ldots, b_{1, m^{\prime}-1}, b_{2, m^{\prime}-1}$ is denoted by $b_{2, \alpha}$. In this case, $B_{\alpha}$ has the form

$$
B_{\alpha}=\left[\begin{array}{cc}
b_{1, \alpha} & b_{3, \alpha} \\
0 & 0
\end{array}\right] \text {. }
$$

Because the previous $B_{j}, j<\alpha$, are nonsingular, we can define all block polynomials $\phi_{0}, \phi_{1}, \ldots, \phi_{\alpha-1}$ by the recurrence relation (4.1). We can not invert $B_{\alpha}$ however. Therefore, we introduce a nonsingular upper triangular matrix $B_{\alpha}^{\prime}$ based on $B_{\alpha}$ by

$$
B_{\alpha}^{\prime}=\left[\begin{array}{cc}
b_{1, \alpha} & b_{3, \alpha} \\
0 & b
\end{array}\right]
$$

with $b$ a free parameter but different from zero. Using this nonsingular matrix $B_{\alpha}^{\prime}$ instead of $B_{\alpha}$ in the recurrence relation, we define $\phi_{\alpha}^{\prime}$ as follows:

$$
\phi_{\alpha}^{\prime}(z) B_{\alpha}^{\prime}=\phi_{\alpha-1}(z)\left(z I_{2}-A_{\alpha}\right)-\phi_{\alpha-2}(z) B_{\alpha-1}^{T} .
$$

It is clear that $\phi_{\alpha}$ has degree $\alpha$ with highest degree coefficient $B_{0}^{-1} B_{1}^{-1} \cdots B_{\alpha-1}^{-1}\left(B_{\alpha}^{\prime}\right)^{-1}$. If we denote the left and right column of $\phi_{\alpha}^{\prime}$ as

$$
\phi_{\alpha}^{\prime}(z)=\left[\begin{array}{ll}
\phi_{\alpha, l}^{\prime}(z) & \phi_{\alpha, r}^{\prime}(z)
\end{array}\right]
$$

we can rewrite the previous equation evaluated in the point $z_{i}$ multiplied to the left by $\left[f_{i}^{\prime},-e_{i}^{\prime}\right]$ as follows

$$
\left[\begin{array}{ll}
f_{i}^{\prime} & -e_{i}^{\prime}
\end{array}\right] \phi_{\alpha, r}^{\prime}\left(z_{i}\right)\left[\begin{array}{ll}
0 & b
\end{array}\right]+Q_{i, \alpha+1}\left[\begin{array}{cc}
b_{1, \alpha} & b_{3, \alpha} \\
0 & 0
\end{array}\right]=Q_{i, \alpha}\left(z I_{2}-A_{\alpha}\right)-Q_{i, \alpha-1} B_{\alpha-1}^{T},
$$

using the fact that $Q_{i, j+1}=\left[f_{i}^{\prime},-e_{i}^{\prime}\right] \phi_{j}\left(z_{i}\right), j=0,1,2, \ldots, \alpha-1$, and the left element of $Q_{i, \alpha+1}$ equals $\left[f_{i}^{\prime},-e_{i}^{\prime}\right] \phi_{\alpha, l}\left(z_{i}\right)$. Because $Q^{T} Z Q=P$, it is easy to see that equation (4.25) reduces to

$$
\left[\begin{array}{ll}
f_{i}^{\prime} & -e_{i}^{\prime}
\end{array}\right] \phi_{\alpha, r}^{\prime}\left(z_{i}\right)=0 .
$$

Hence, we immediately conclude that $n^{\prime}(z) / d^{\prime}(z)$ with

$$
\left[\begin{array}{l}
d^{\prime}(z) \\
n^{\prime}(z)
\end{array}\right]=\phi_{\alpha, r}^{\prime}(z)
$$

approximates the given data exactly, i.e. $n^{\prime}(z) / d^{\prime}(z)$ is an interpolant of the given data. It is clear that this situation will appear rarely in practice when the data are only given with a certain finite precision. However, we shall give an example of this situation in one of the next sections. It is clear that we can always normalize the interpolant $n^{\prime}(z) / d^{\prime}(z)$ such that $n_{\alpha}^{\prime}=1$, because we know the highest degree coefficient of $\phi_{\alpha}^{\prime}(z)$.

\section{The description of an elementary step of the algo- rithm}

Let us investigate the reduction scheme in more detail. Suppose we are computing row and column $l$ of the pentadiagonal matrix $P$ and we have already shifted the nonzero elements 
$k-1$ places to the right and, $k-1$ places down. In other words, we have the following situation.

$$
\begin{array}{ccccccccc}
\ddots & \ddots & \ddots & c_{k} & & & & & t \\
\ddots & \ddots & \ddots & b_{k} & \ddots & & & & u \\
& c_{k} & b_{k} & a_{k} & b_{k+1} & c_{k+2} & & & v \\
& & \ddots & b_{k+1} & \ddots & \ddots & \ddots & & w \\
& & & c_{k+2} & \ddots & \ddots & \ddots & \ddots &
\end{array}
$$

$$
t \quad u \quad v \quad w \quad r
$$

We have only indicated those entries playing a role in shifting the nonzero elements in the $l$ th row one place to the right and in the $l$ th column one place down. First of all, we apply the Givens reflection $G_{l, k}$ to the $k$ th and $l$ th row such that

$$
G_{l, k}\left[\begin{array}{c}
c_{k} \\
t
\end{array}\right]=\left[\begin{array}{c}
c_{k}^{\prime \prime} \\
0
\end{array}\right]
$$

We obtain the following situation:

$$
\begin{array}{ccccccccc}
\ddots & \ddots & \ddots & c_{k} & & & & & t \\
\ddots & \ddots & \ddots & b_{k} & \ddots & & & & u \\
& c_{k}^{\prime \prime} & b_{k}^{\prime \prime} & a_{k}^{\prime} & b_{k+1}^{\prime \prime} & c_{k+2}^{\prime \prime} & & & u^{\star} \\
& & \ddots & b_{k+1} & \ddots & \ddots & \ddots & & w \\
& & & c_{k+2} & \ddots & \ddots & \ddots & \ddots &
\end{array}
$$

$$
0 \quad t^{\prime \prime} \quad u^{\prime} \quad v^{\prime \prime} \quad w^{\prime \prime} \quad r^{\prime}
$$

Applying the Givens reflection $G_{l, k}^{T}=G_{l, k}$ to the $k$ th and $l$ th column gives us the next scheme.

$$
\begin{array}{ccccccccc}
\ddots & \ddots & \ddots & c_{k}^{\prime \prime} & & & & & 0 \\
\ddots & \ddots & \ddots & b_{k}^{\prime \prime} & \ddots & & & & t^{\prime \prime} \\
& c_{k}^{\prime \prime} & b_{k}^{\prime \prime} & a_{k}^{\prime \prime} & b_{k+1}^{\prime \prime} & c_{k+2}^{\prime \prime} & & & u^{\prime \prime} \\
& & \ddots & b_{k+1}^{\prime \prime} & \ddots & \ddots & \ddots & & v^{\prime \prime} \\
& & & c_{k+2}^{\prime \prime} & \ddots & \ddots & \ddots & \ddots &
\end{array}
$$

$$
0 \quad t^{\prime \prime} \quad u^{\prime \prime} \quad v^{\prime \prime} \quad w^{\prime \prime} \quad r^{\prime \prime}
$$

If we drop the double quotes, we get the notation of the initial situation. If $k+1<l$, we can increment $k$ and repeat the computational step, i.e. apply a Givens reflection to the left and right. If $k+1=l$, we handle the next row, if there is one, i.e. we increment $l$ and set $k$ equal to one. 
Let us summarize how the entries can be computed:

$$
\left[\begin{array}{cccccc}
c_{k}^{\prime \prime} & b_{k}^{\prime \prime} & a_{k}^{\prime} & b_{k+1}^{\prime \prime} & c_{k+2}^{\prime \prime} & u^{\star} \\
0 & t^{\prime \prime} & u^{\prime} & v^{\prime \prime} & w^{\prime \prime} & r^{\prime}
\end{array}\right]=G_{l, k}\left[\begin{array}{cccccc}
c_{k} & b_{k} & a_{k} & b_{k+1} & c_{k+2} & v \\
t & u & v & w & 0 & r
\end{array}\right]
$$

and

$$
\left[\begin{array}{cc}
a_{k}^{\prime \prime} & u^{\prime \prime} \\
u^{\prime \prime} & r^{\prime \prime}
\end{array}\right]=\left[\begin{array}{cc}
a_{k}^{\prime} & u^{\star} \\
u^{\prime} & r^{\prime}
\end{array}\right] G_{l, k}
$$

Note that we do not need to compute $w^{\prime \prime}$ and $c_{k+2}^{\prime \prime}$ if $k=l-2$ or $k=l-1$. If $k=l-1$, we also do not have to compute $v^{\prime \prime}$ and $b_{k+1}^{\prime \prime}$.

\section{$6 \quad$ Pseudomonic polynomials}

Instead of computing the block orthogonal polynomials $\phi_{j}(z)$ and then normalizing the corresponding polynomial couples, we can immediately compute the pseudomonic block orthogonal polynomials $\psi_{j}(z)$, defined as follows

Definition 6.1 (pseudomonic block orthogonal polynomials) The pseudomonic orthogonal polynomials $\psi_{j}(z)$ are related to the orthonormal polynomials $\phi_{j}(z)$ (if they exist) by

$$
\psi_{j}(z)=\phi_{j}(z)\left[\begin{array}{cc}
\prod_{k=1}^{j+1} c_{2 k-1} & 0 \\
0 & \prod_{k=1}^{j+1} c_{2 k}
\end{array}\right]
$$

where $c_{2 k-1}$ and $c_{2 k}$ are the diagonal elements of $B_{k-1}$, i.e. $c_{2 k-1}=b_{1, k-1}$ and $c_{2 k}=b_{2, k-1}$ from (4.12). In other words, the highest degree coefficient of $\psi_{j}(z)$ is a unit upper triangular matrix.

The pseudomonic orthogonal polynomials satisfy the recurrence given in the following theorem.

Theorem 6.1 The pseudomonic block orthogonal polynomials $\psi_{j}(z)$ can be defined by the recurrence relation

$$
\psi_{j}(z)=\psi_{j-1}(z)\left(z D_{j}-E_{j}\right)-\psi_{j-2}(z) F_{j}, \quad j=1,2, \ldots, m^{\prime}-1,
$$

with the initialization

$$
\psi_{-1}=\left[\begin{array}{ll}
0 & 0 \\
0 & 0
\end{array}\right] \text { and } \psi_{0}=\left[\begin{array}{cc}
1 & -b_{1} / c_{1} \\
0 & 1
\end{array}\right] .
$$

The matrices $D_{j}, E_{j}, F_{j}$ appearing in the recurrence relation are defined as follows:

$$
\begin{aligned}
D_{j} & =\left[\begin{array}{cc}
1 & d_{j} q_{j} \\
0 & 1
\end{array}\right], \\
E_{j} & =\left[\begin{array}{cc}
a_{2 j-1} & \left(a_{2 j-1} d_{j}+b_{2 j}\right) q_{j} \\
b_{2 j} / q_{j} & b_{2 j} d_{j}+a_{2 j}
\end{array}\right], \\
F_{j} & =\left[\begin{array}{cc}
u_{j} & d_{j} u_{j} q_{j} \\
h_{j} / q_{j} & d_{j} h_{j}+g_{j}
\end{array}\right]
\end{aligned}
$$

with

$$
d_{j}=-b_{2 j+1} / c_{2 j+1}, \quad u_{j}=c_{2 j-1}^{2}, \quad h_{j}=b_{2 j-1} c_{2 j}, \quad g_{j}=c_{2 j}^{2} \text { and } q_{j}=\prod_{k=1}^{j} c_{2 k} / \prod_{k=1}^{j} c_{2 k-1} .
$$


Proof. It is easy to prove this by induction using the recurrence relation (4.1) for the $\phi_{j}(z)$, knowing that

$$
A_{j}=\left[\begin{array}{cc}
a_{2 j-1} & b_{2 j} \\
b_{2 j} & a_{2 j}
\end{array}\right] \text { and } B_{j}=\left[\begin{array}{cc}
c_{2 j+1} & b_{2 j+1} \\
0 & c_{2 j+2}
\end{array}\right]
$$

Note that we have included "if they exist" in the definition. The value of $c_{2 j+1}$ does not play a role when computing the left column of $\psi_{j}(z)$. Similarly the value of $c_{2 j+2}$ does not appear when deriving the right column of $\psi_{j}(z)$. Therefore, the pseudomonic orthogonal polynomials $\psi_{j}(z)$ can be defined based on $\phi_{j}(z)$ taking the limit when either $c_{2 j+1}$ or $c_{2 j+2}$ is zero, i.e. when we have a rational interpolant. Using the recurrence relation for the pseudomonic block orthogonal polynomials $\psi_{j}(z)$, we can easily derive that

$$
\left[\begin{array}{ll}
\psi_{\beta-1}(z) & \psi_{\beta}(z)
\end{array}\right]=\left[\begin{array}{ll}
\psi_{-1}(z) & \psi_{0}(z)
\end{array}\right] V_{1} V_{2} \cdots V_{\beta}
$$

with

$$
V_{j}=\left[\begin{array}{cc}
0 & -F_{j} \\
I & z D_{j}-E_{j}
\end{array}\right]
$$

The solution $\sigma=[d n]^{T}$ is given by $\left[\psi_{\beta}\right] t$ with $t=\left[\begin{array}{ll}1 & 0\end{array}\right]^{T}$ or $\left[\begin{array}{ll}0 & 1\end{array}\right]^{T}$ depending on whether we choose the "left" or the "right" structure.

\section{Forward/backward evaluation}

To compute the coefficients as well as function values of the rational approximant, we can use a forward or a backward recurrence scheme. For example, the backward recurrence scheme to compute $\sigma=[d n]^{T}$ works as follows. If we have chosen the "left" structure, we initialize $\sigma_{\beta+1}$ as

$$
\sigma_{\beta+1}=\left[\begin{array}{llll}
0 & 0 & 1 & 0
\end{array}\right]^{T},
$$

and, if we have chosen the "right" structure, we start with

$$
\sigma_{\beta+1}=\left[\begin{array}{llll}
0 & 0 & 0 & 1
\end{array}\right]^{T} \text {. }
$$

With this initialization, we use the backward recurrence scheme

$$
\sigma_{j}=V_{j} \sigma_{j+1}, \quad j=\beta, \beta-1, \ldots, 1
$$

with $V_{j}$ as defined above and with the final step

$$
\sigma=\left[\begin{array}{l}
d(z) \\
n(z)
\end{array}\right]=\left[\begin{array}{ll}
\psi_{-1} & \psi_{0}
\end{array}\right] \sigma_{1} .
$$

\section{Parallel implementation}

Regarding the amount of computational work, we can make a distinction between the computation of the block tridiagonal matrix $P$ from the data and the computation of the orthonormal block polynomials. Fixing the degree $\alpha \ll m$ of the rational approximant implies that we can obtain the rational approximant from $\phi_{\alpha}(z)$. Hence, it is not necessary to compute the complete pentadiagonal matrix $P$ but only the first part consisting of $A_{1}, A_{2}, \ldots, A_{\alpha}$ and 
$B_{1}, B_{2}, \ldots, B_{\alpha}$. Compared to the complexity $m^{2}$ of computing the complete block tridiagonal matrix $P$, this decreases the amount of computational work to $m \alpha$. The second part of the solution method requires the computation of $\phi_{0}, \phi_{1}, \ldots, \phi_{\alpha}$, which requires a number of floating point operations of the order of $\alpha^{2}$. Therefore, since $\alpha \ll m$, it is important to decrease the execution time of the first part by parallel implementation. We have tried several scenarios. For the divide and conquer approach, we could not find an efficient way to join the solutions of two subproblems. The parallel approach, that we implemented, is a sort of pipeline method.

Let us assume that we have $N<m$ processors in the reduction scheme of the previous section. The $n$th processor will handle rows $n+1+j \cdot N, j=0,1, \ldots,[(m-n-1) / N]$, where [.] denotes the integer part. These $N$ processors are synchronized in a pipeline manner. Suppose the $n$th processor is executing the Givens reflection to the left and right on the $l$ th and $k$ th row and column by using the formulas above. As soon as $c_{k}^{\prime \prime}$ is computed, the $(n+1)$ st processor could use this value for handling the $(l+1)$ st and $(k-2)$ nd row and column. Therefore, the $n$th processor has to compute $c_{k}^{\prime \prime}$ as soon as possible, while the $(n+1)$ st processor has to delay the use of $c_{k}^{\prime \prime}$ as much as possible. We have not used this fine tuned synchronization in the implementation. Instead, we let the $(n+1)$ st processor start on row and column $k+2$ as soon as the $n$th processor has computed the value $c_{k}^{\prime \prime}$. The influence on the execution time can be seen as a difference in startup time of the pipeline. Neglecting the synchronization time, we thus obtain a reduction of the computation time by a factor $N$ when using $N$ processors. Because we only have to know $A_{1}, A_{2}, \ldots, A_{\alpha}$ and $B_{0}, B_{1}, B_{2}, \ldots, B_{\alpha}$, it is not necessary to shift each row and column completely to the diagonal. From the $(\alpha+2)$ nd block row on, we have to shift only over $(\alpha+1)$ block places. The elements $c_{1}, c_{2}, \ldots, c_{2 \alpha+2}$ will determine the approximation error, i.e. the distance between the "left" or "right" structure and the data. After the execution of the first step of the algorithm, we shall print the products $\prod_{j=1}^{\beta+1} c_{2 j-1}$ and $\prod_{j=1}^{\beta+1} c_{2 j}$ for $\beta=0,1,2, \ldots, \alpha$. Given these approximation errors, the user chooses an approximation degree $\beta \leq \alpha$ and the "left" or "right" structure for the approximant. As we have shown in subsection 4.2 , the first zero entry in this sequence of approximation errors will indicate a rational interpolant. In practice, however, a value exactly equal to zero as in (4.21) will never be attained.

\section{$9 \quad$ Examples}

In this section, we give two examples. The first one is rather theoretical, the second one is more practical. To measure the goodness of the rational approximant, we consider a relative and absolute approximation error in each point $z_{i}$. If $\operatorname{abs}\left(e_{i}\right) \geq \operatorname{abs}\left(f_{i}\right)$, the absolute error is defined as $\operatorname{abserr}\left(z_{i}\right)=\operatorname{abs}\left(\left(n\left(z_{i}\right) / d\left(z_{i}\right)\right)-\left(f_{i} / e_{i}\right)\right)$ (if $\left.d\left(z_{i}\right) \neq 0\right)$ and the relative error as relerr $\left(z_{i}\right)=\operatorname{abs}\left(\operatorname{abserr}\left(z_{i}\right) /\left(f_{i} / e_{i}\right)\right)$ if $\left.f_{i} / e_{i} \neq 0\right)$. If $\operatorname{abs}\left(e_{i}\right)<\operatorname{abs}\left(f_{i}\right)$, the absolute error is defined as abserr $\left(z_{i}\right)=\operatorname{abs}\left(\left(d\left(z_{i}\right) / n\left(z_{i}\right)\right)-\left(e_{i} / f_{i}\right)\right)$ (if $\left.n\left(z_{i}\right) \neq 0\right)$ and the relative error as $\operatorname{relerr}\left(z_{i}\right)=\operatorname{abs}\left(\operatorname{abserr}\left(z_{i}\right) /\left(e_{i} / f_{i}\right)\right.$ ) (if $e_{i} / f_{i} \neq 0$ ). The value of the rational approximant $n\left(z_{i}\right) / d\left(z_{i}\right)$ or the inverse is computed using the Horner scheme. Of course, we could also use the backward recurrence where $z$ is replaced by $z_{i}$. Besides using weights, it is possible to scale the interpolation data such that $\max \left\{\operatorname{abs}\left(f_{i}\right), \operatorname{abs}\left(e_{i}\right)\right\}=1$. For the examples which we tried, the scaling did not improve the results. All computations were done in double precision on an IBM3090 using parallel FORTRAN.

Example 9.1 In the first example, we consider the 10 interpolation points $z_{i}=i, i=$ $1,2, \ldots, 10$, with function values $f_{i} / e_{i}$, where $f_{i}=i+1$ and $e_{i}=i+2$. The weights are set equal to one. We assume all the quantities that are given as input to the parallel FORTRAN 


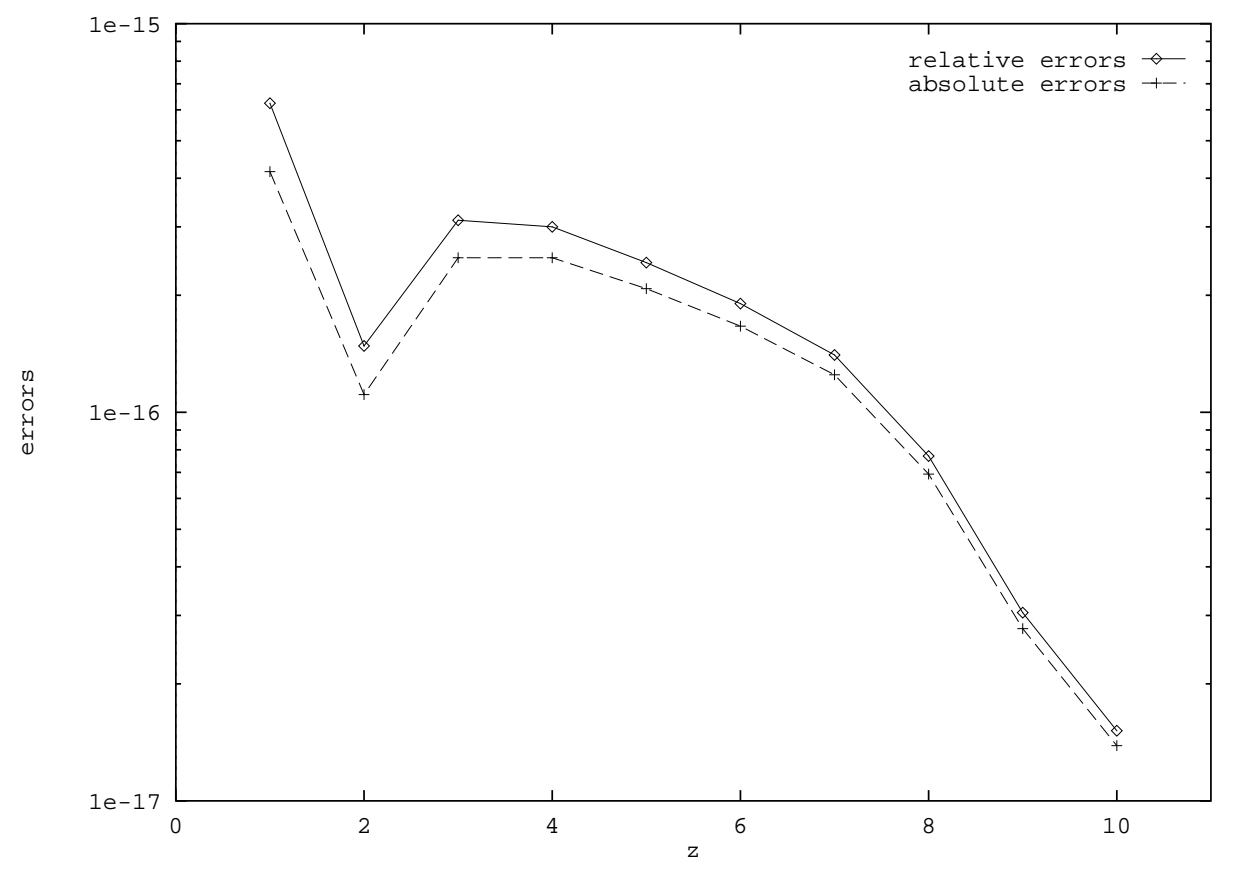

Figure 1: The absolute and relative errors of example 1

program are known within machine precision. With $\alpha$ chosen as big as possible, i.e. equal to 4 , we get the following table of approximation errors.

\begin{tabular}{|r|r|r|}
\hline$j$ & $\prod_{k=0}^{J} c_{2 k+1}$ & $\prod_{k=0}^{J} c_{2 k+2}$ \\
\hline 0 & $0.2540 D+02$ & $0.1131 D+01$ \\
1 & $0.2298 D+02$ & $-0.1513 D-13$ \\
2 & $0.4326 D+02$ & $-0.2085 D-13$ \\
3 & $-0.8152 D+02$ & $0.1284 D-13$ \\
4 & $0.1084 D+03$ & $-0.1756 D-13$ \\
\hline
\end{tabular}

From this table, it is clear that we should choose $\beta$ equal to 1 and take the "right" structure for the rational approximant. The next table shows the coefficients of the numerator and denominator polynomial of the rational approximant of degree $\beta=1$.

\begin{tabular}{|c|c|c|}
\hline degree & numerator coefficients & denominator coefficients \\
\hline 0 & $0.9999999999999806 D+00$ & $0.1999999999999972 D+01$ \\
1 & $0.9999999999999993 D+00$ & $0.1000000000000000 D+01$ \\
\hline
\end{tabular}

The absolute and relative errors are indicated in Figure 1. We can say that the rational approximant, we obtained here, is very good, which, for this example of course will not come as a surprise.

A more realistic example is the following one.

Example 9.2 We are starting from the rational function

$$
g(z)=\frac{100 z^{4}+10520 z^{3}+52101 z^{2}+10105 z+500}{8169.13 z^{3}+50664.97 z^{2}+9984.32 z+500} .
$$

The inverse of this function was used to demonstrate model reduction techniques [2, 9]. The data points are $z_{i}=i / 10, i=1,2, \ldots, 100$. The function values are given by $f_{i}=g\left(z_{i}\right)$, 
Figure 2: The absolute and relative errors of example 2

computed by the algorithm of Horner, and $e_{i}=1.0$. The weights are taken equal to one. For $\alpha=10$, we get the following table of approximation errors.

\begin{tabular}{|r|r|r|}
\hline$j$ & $\prod_{k=0}^{\jmath} c_{2 k+1}$ & $\prod_{k=0}^{\jmath} c_{2 k+2}$ \\
\hline 0 & $0.1000 D+02$ & $0.6786 D+00$ \\
1 & $0.3736 D+01$ & $0.3058 D+00$ \\
2 & $0.1824 D+01$ & $0.4317 D+00$ \\
3 & $-0.7181 D-06$ & $0.8036 D+00$ \\
4 & $-0.1084 D-09$ & $0.1942 D+01$ \\
5 & $-0.1142 D-09$ & $0.4571 D+01$ \\
6 & $-0.1957 D-09$ & $0.1137 D+02$ \\
7 & $-0.3880 D-09$ & $0.2790 D+02$ \\
8 & $-0.9079 D-09$ & $0.6308 D+02$ \\
9 & $-0.1937 D-08$ & $0.1600 D+03$ \\
10 & $-0.4190 D-08$ & $0.3977 D+03$ \\
\hline
\end{tabular}

From these rational approximation errors, it is clear that we should use a "left" structure. First of all, we choose $\beta$ equal to 4 . The coefficients of the rational approximant are given in the next table.

\begin{tabular}{|c|c|c|}
\hline degree & numerator coefficients & denominator coefficients \\
\hline 0 & $0.4999659362429158 D+01$ & $0.4999659360979468 D+01$ \\
1 & $0.1010465049127602 D+03$ & $0.9983978711954701 D+02$ \\
2 & $0.5210092803518180 D+03$ & $0.5066491406348295 D+03$ \\
3 & $0.1051999931526788 D+03$ & $0.8169129999998811 D+02$ \\
4 & $0.1000000000000000 D+01$ & \\
\hline
\end{tabular}

The absolute and relative approximation errors in each data point are shown in Figure 2. If we choose $\beta$ equal to 3 , we get the following table of coefficients.

\begin{tabular}{|c|c|c|}
\hline degree & numerator coefficients & denominator coefficients \\
\hline 0 & $0.4974901332730807 D+02$ & $0.4974902323831709 D+02$ \\
1 & $0.5104473551313152 D+03$ & $0.4984396214923242 D+03$ \\
2 & $0.1050995010534305 D+03$ & $0.8169130146942339 D+02$ \\
3 & $0.1000000000000000 D+01$ & \\
\hline
\end{tabular}

Figure 3 indicates the absolute and relative error of this rational approximant in each data point. Considering the data points in reverse order gives the same results. As a conclusion, we can say that the original rational function $g(z)$ can be approximated very well by a rational approximant of degree 3 , as was already known in the model reduction context.

\section{Conclusion}

We have developed an efficient method to solve the discrete least squares rational approximation problem. We have shown that this method can be implemented in a pipelined way on a parallel architecture.

Because the algorithm developed here is so efficient, it could be used as the kernel of a graphical interactive program to design rational approximants. As domains of application, we mention digital filter design, modal analysis, model reduction, system identification,.... 


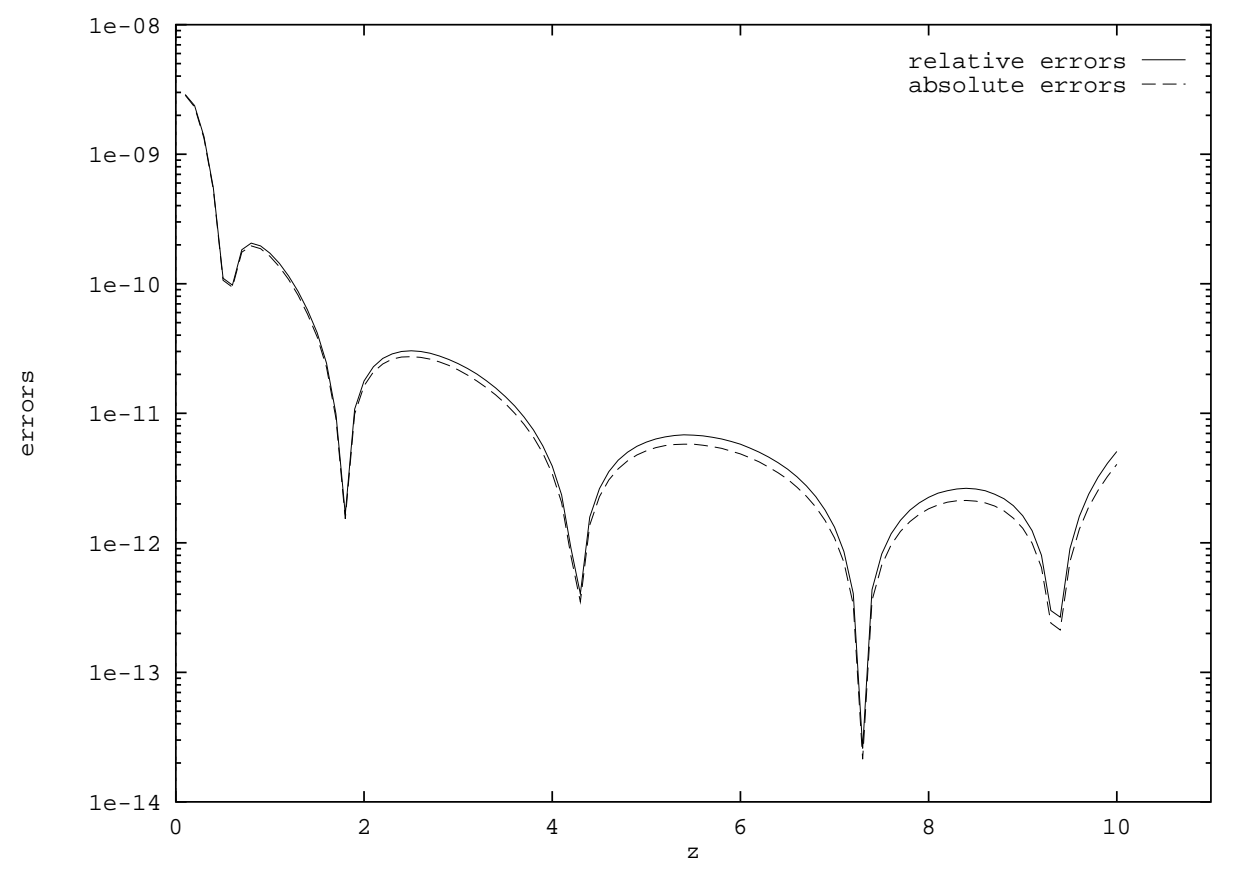

Figure 3: The absolute and relative errors of example 2 with $\beta=3$

To enhance numerical stability, we have already mentioned that the backward recurrence scheme can also be used to evaluate the rational approximant. A compromise between using the coefficients for the classical basis $1, z, z^{2}, \ldots$ and the backward recurrence scheme, would be to compute the coefficients of the numerator and denominator polynomial of the rational approximant with respect to a more "orthogonal" basis on the approximation interval, e.g. with respect to the Chebyshev polynomials. This is easy as long as the multiplication by $z$ (the shift operation) of such a basis polynomial can be done in an efficient way. Note that we can do this efficiently for orthogonal polynomials using the three term recurrence relation.

To summarize, we have constructed a method, which is so efficient that it can be used interactively on large data sets.

\section{References}

[1] G.S. Ammar and W.B. Gragg. $o\left(n^{2}\right)$ reduction algorithms for the construction of a band matrix from spectral data. SIAM J. Matrix Anal. Appl., 12:426-431, 1991.

[2] R. K. Appiah. Linear model reduction using Hurwitz polynomial approximation. Int. J. Contr., 28(3):467-488, 1978.

[3] D.L. Boley and G.H. Golub. A survey of matrix inverse eigenvalue problems. Physics Trust Publications, 3:595-622, 1987.

[4] S. Elhay, G.H. Golub, and J. Kautsky. Updating and downdating of orthogonal polynomials with data fitting applications. SIAM J. Matrix Anal. Appl., 12:327-353, 1991.

[5] W. B. Gragg and W. J. Harrod. The numerically stable reconstruction of Jacobi matrices from spectral data. Numer. Math., 44:317-335, 1984. 
[6] L. Reichel. Fast QR decomposition of Vandermonde-like matrices and polynomial least squares approximation. SIAM J. Matrix Anal. Appl., 12:552-564, 1991.

[7] L. Reichel, G.S. Ammar, and W.B. Gragg. Discrete least squares approximation by trigonometric polynomials. Math. Comp., 57:273-289, 1991.

[8] H. Rutishauser. On Jacobi rotation patterns. In Proceedings of Symposia in Applied Mathematics, vol. 15, Experimental Arithmetic, High Speed Computing and Mathematics, pages 219-239, Providence, 1963. Amer. Math. Society.

[9] Y. Shamash. Viability of methods for generating stable reduced order models. IEEE Trans. on Automatic Control, AC-26(6):1285-1286, December 1981.

[10] M. Van Barel and A. Bultheel. A new approach to the rational interpolation problem. J. Comp. Appl. Math., 32:281-289, 1990.

[11] M. Van Barel and A. Bultheel. A new approach to the rational interpolation problem: the vector case. J. Comp. Appl. Math., 33:331-346, 1990. 